\title{
Composite group of explicit Runge-Kutta methods
}

\begin{abstract}
In this paper,the composite groups of Runge-Kutta (RK) method are proposed. The composite group of RK method of third and second order, RK3(2) and fourth and third order RK4(3) base on classical Runge-Kutta method are derived. The proposed methods are twostep in nature and have less number of function evaluations compared to the existing RungeKutta method. The order conditions up to order four are obtained using rooted trees and composite rule introduced by J. C Butcher. The stability regions of RK3(2) and RK4(3) methods are presented and initial value problems of first order ordinary differential equations are carried out. Numerical results are compared with existing Runge-Kutta method.
\end{abstract}

Keyword: Composite groups; Runge-Kutta methods 